\title{
hMICL and CD123 in combination with a CD45/CD34/CD117 backbone - a universal marker combination for the detection of minimal residual disease in acute myeloid leukaemia
}

\author{
Anne S. Roug, ${ }^{1}$ Hanne $\emptyset$. Larsen, ${ }^{1}$ Line \\ Nederby, ${ }^{1}$ Tom Just, ${ }^{2}$ Gordon Brown, ${ }^{3}$ \\ Charlotte G. Nyvold, ${ }^{1}$ Hans B. Ommen ${ }^{1}$ \\ and Peter Hokland ${ }^{1}$ \\ ${ }^{1}$ Department of Haematology, Aarhus University \\ Hospital, Aarhus, ${ }^{2}$ Dako, Glostrup, Denmark \\ and ${ }^{3}$ Section of Infection and Immunity, Insti- \\ tute of Medical Sciences, University of Aberdeen, \\ Aberdeen, UK
}

Received 20 June 2013; accepted for publication 6 September 2013

Correspondence: Peter Hokland, Aarhus University Hospital, Department of Haematology, Tage-Hansens Gade 2, DK-8000 Aarhus C, Denmark.

E-mail: phokland@ki.au.dk

\begin{abstract}
Summary
Real-time quantitative polymerase chain reaction (qPCR) has been extensively validated for the detection of minimal residual disease (MRD) in acute myeloid leukaemia (AML). Meanwhile, multicolour flow cytometry (MFC) has received less attention because the so-called leukaemia-associated immunophenotypes (LAIPs) are generally of lower sensitivity and specificity, and prone to change during therapy. To improve MRD assessment by MFC, we here evaluate the combination of human Myeloid Inhibitory C-type Lectin (hMICL, also termed C-type lectin domain family 12, member A, CLEC12A) and CD 123 (also termed interleukin-3 receptor alpha, IL3RA) in combination with CD34 and CD117 (KIT), as an MRD assay in pre-clinical and clinical testing in 69 AML patients. Spiking experiments revealed that the assay could detect MRD down to $10^{-4}$ in normal bone marrow with sensitivities equalling those of validated $\mathrm{qPCR}$ assays. Moreover, it provided at least one MFC MRD marker in 62/69 patients (90\%). High levels of hMICL/ CD123 LAIPs at the post-induction time-point were a strong prognostic marker for relapse in patients in haematological complete remission $(P<0.001)$. Finally, in post induction samples, hMICL/CD123 LAIPs were strongly correlated $(r=0.676, P=0.0008)$ to applied qPCR targets. We conclude the hMICL/CD123-based MFC assay is a promising MRD tool in AML.
\end{abstract}

Keywords: acute myeloid leukaemia, flow cytometry, minimal residual disease, quantitative polymerase chain reaction, leukaemia-associated immunophenotype.
The majority of curatively treated acute myeloid leukaemia (AML) patients will experience a relapse, although deemed being in complete remission (CR) by standard diagnostic criteria (Estey \& Döhner, 2006). This is caused by the presence and outgrowth of sustaining leukaemic stem cells (LCSs) in the bone marrow (BM), termed minimal residual disease (MRD) (Bachas et al, 2012). Although the translation of some MRD assays into therapeutic decision-making has been accomplished, especially in acute lymphoblastic leukaemia (ALL), routinely prospective risk stratification in AML based on MRD monitoring is marred by lack of standardized assays and thresholds. Additionally, formal proof supporting preemptive intervention of MRD-positive AML patients is still sparse (Paietta, 2012a,b; Vora et al, 2013).

The real-time quantitative polymerase chain reaction (qPCR) is the most sensitive and specific molecular MRD detection method in AML, employing fusion transcripts and over-expressed and mutated genes with sensitivities ranging from $10^{-2}$ to $10^{-6}$ and is applicable in approximately $60 \%$ of patients (Grimwade et al, 2009, 2010a; Hokland et al, 2012). By contrast, MRD detection by multicolour flow cytometry (MFC) is faster, determines accurately the number of leukaemic cells, and is applicable to virtually all AML patients with sensitivities ranging from $10^{-2}$ to $10^{-4}$ in most cases (Buccisano et al, 2012; Dinardo \& Luger, 2012). The MFC strategy relies upon detection of so-called leukaemia-associated immunophenotypes (LAIPs), which are absent or only rarely present in normal BM. Thus, advantage is taken of aberrations in antigen expression, such as lack of expression, cross-lineage expression, over-expression, and asynchronous expression, usually in combination with abnormal light scatter patterns of leukaemic cells (Al-Mawali et al, 2008; Ossenkoppele et al, 
2011). This approach, however, requires the availability of a comprehensive panel of antibodies at the initial diagnosis for identification of optimal LAIP combinations. In addition, the heterogeneity of AML cells within each patient sample can cause a selective shift in immunophenotype during cytoreduction, which may yield false negative MRD results (Kern et al, 2010a). Moreover, LAIPs are usually identified by the expression of CD34 on leukaemic cells, even though up to $30 \%$ of AML cases are CD34- (Bonnet, 2001; Feller et al, 2004; Al-Mawali et al, 2009). A novel approach within MRD assessment by MFC aims at enumerating LSCs rather than the whole blast population by quantification of aberrant antigen expression on $\mathrm{CD}^{+} 4^{+} \mathrm{CD} 38$ - stem cells (van Rhenen et al, 2007a). However, this approach also fails in cases of CD34- AML as the remaining CD34 + CD38- cells in such patients are usually of normal origin (van der Pol et al, 2003; Taussig et al, 2010).

Recently, we have identified the human Myeloid Inhibitory C type-like Lectin (hMICL) (also known as CLEC12A or CLL-1) as a stable and reliable AML antigen expressed in 90\% of diagnostic AML samples (Larsen et al, 2012). Additionally, we found that CD123 (also known as interleukin $3 \alpha$ receptor, IL3RA) is widely, but not exclusively, expressed in AML. Importantly, both hMICL and CD123 had high and conserved antigen expression in 23/23 paired diagnostic/ relapse samples, confirming that antigenic shift is, at most, a minor problem with these antigens (Larsen et al, 2012). Finally, hMICL and CD123 have been reported to be robust markers for LSCs and thus possible targets in antibody mediated anti-leukaemia treatment (van Rhenen et al, 2005, 2007b; Majeti, 2010).

In this study we have evaluated the MRD potential of a single tube MFC experiment employing hMICL and CD123 in combination with CD45 (also known as PTPRC), CD34, CD117 (also known as KIT), and CD14. The rationale for employing CD45, CD34, and CD117 is that these antigens have proven the most frequent backbone markers in AML and consequently included the add on of hMICL and CD123 (Al-Mawali et al, 2009; van Dongen et al, 2012). We initially performed a series of paralleled MFC and qPCR spiking experiments and then tested the assay in a prospective study encompassing 69 patients. Taken together, this data set shows that the hMICL/CD123 MFC assay is a valuable addition to MRD monitoring with sensitivities approaching those obtained by qPCR assays in a simplified MFC-based setting covering more than $90 \%$ of AML patients.

\section{Materials and methods}

\section{Patient samples}

All sampling was conducted with informed patient consent and in accordance with the Declaration of Helsinki. The study was approved by the local Ethics Committee. A total of 69 prospectively accrued diagnostic AML samples were obtained from the Department of Haematology, Aarhus
University Hospital, from June 2009 to June 2011. A total of 40 patients were treated with curative intent. Of these, 21 patients were evaluable for post-induction response by paralleled MFC and qPCR MRD quantification (Table I). Normal $\mathrm{BM}$ from healthy volunteers $(n=12)$ and regenerating $\mathrm{BM}$ from ALL patients $(n=7)$ obtained at day 29 were used as control samples for sensitivity studies and for generation of internal standard references. The ALL patients included were all diagnosed at our institution and enrolled in the NOPHO 2008 study. In this protocol, patients were systematically subjected to response evaluation post-induction at day 29. Normal donor $\mathrm{CD}^{+}$T-cells for spiking experiments were isolated from peripheral blood from two healthy donors using the immunomagnetic EasySep CD3 Positive Selection Kit (StemCell Technologies Grenoble, France).

\section{Flow cytometry}

Standard four-colour MFC was performed on erythrocytelysed whole BM with monoclonal antibody (MoAb) combinations for diagnostic purpose, LAIP identification and monitoring of MRD in follow-up samples. An additional

Table I. Patient characteristics.

\begin{tabular}{ll}
\hline Characteristic & Value \\
\hline Sex, number of patients (male/female) & $69(41 / 28)$ \\
Age, years (median; range) & $65 \cdot 5(7-94)$ \\
Total leucocytes x $10^{9} / 1$ (median; range) & $43(0 \cdot 1-312)$ \\
Bone marrow blasts at diagnosis\% (median; range) & $58(20-97)$ \\
Karyotype $(n)^{*}$ & \\
Favourable & 4 \\
Unfavourable & 11 \\
Intermediate & 47 \\
Not done & 7 \\
Molecular MRD target & \\
WT1 & 36 \\
PML-RARA & 1 \\
RUNX1-RUNX1T1 & 2 \\
CBFB-MYH11 & 1 \\
Negative & 8 \\
Not done (age > 70 years) & 22 \\
CD34 expression & \\
CD34- & 18 \\
CD34+ & 41 \\
Treated with curative intent & 40 \\
Available paired MFC/qPCR samples post induction & 21 \\
\hline
\end{tabular}

MRD, minimal residual disease; MFC, multicolour flow cytometry; qPCR, real-time quantitative polymerase chain reaction.

*'Favourable', cases with $\mathrm{t}(8 ; 21), \mathrm{t}(15 ; 17)$, or inv(16)/t(16;16); 'unfavourable', cases with complex cytogenetic changes $(>3$ unrelated abnormalities), $-5, \operatorname{add}(5 q) / \operatorname{del}(5 q),-7 / \operatorname{add}(7 q), t(6 ; 11), t(10 ; 11)$, $\mathrm{t}(9 ; 22),-17, \operatorname{abn}(17 \mathrm{p})$ with other changes, $3 \mathrm{q}$ abnormalities excluding $t(3 ; 5)$, inv(3)/t(3;3); and 'intermediate', cases with normal karyotype and other noncomplex. (Grimwade et al, 2010b).

${ }^{*}$ Applying a cut off of $1 \% \mathrm{CD} 34+$ blast cells. 
tube with a six-colour combination including MoAbs against hMICL, CD123, CD45, CD34, CD117 and CD14 was run in all cases. All combinations of MoAbs and their purveyors are given in Table S1. Data acquisition was performed on a Canto II [BD Biosciences, San Jose, CA, USA (BD)] and analysed using BD FACSDiva software (BD). Apoptotic and dead cells were deselected using Annexin V and 7-Aminoactinomycin D (7-AAD; BD). A minimum of 250000 live $\mathrm{CD} 45^{+}$events was acquired, resulting in a theoretical maximum sensitivity of at least $0 \cdot 008 \%$ in these experiments taking into account that a cluster of 20 events is needed for clear definition of a cell population (Buccisano et al, 2009).

\section{Identification of LAIPs, calculations based on MFC analyses and Gating Strategy}

Leukaemic cells were identified based on low expression of CD45 and low side scatter (SSC) properties (CD45low/ SSClow) (Borowitz et al, 1993; Lacombe et al, 1997). As differentiated monocytes express both hMICL and CD123, CD14 de-selection was performed except for cases with CD14+ AML. Potential LAIP populations were identified by the expression of combinations of hMICL, CD123, CD34, and CD117 and quantified in normal and leukaemic BM, applying the same stringent gating strategy in all samples. A threshold of at least 5\% LAIP positive cells was set for a given LAIP combination to be denoted as positive. All LAIP positive cells were back-gated to FSC/SSC plots to ensure homogeneous scatter properties. Comparisons between AML and control samples allowed for the discovery of an 'empty space', defined as regions devoid of normal BM cells in an hMICL/CD123 dot plot. The gating strategy used and representative examples of LAIPs are depicted in Fig 1.

In order to estimate the degree of reduction in leukaemic mass detectable by the present approach, the logarithmic difference (LD) between AML and normal BM samples was calculated as the frequency of a LAIP in AML BM divided by that of the LAIP in normal and regenerating BM. The maximal LD observed was used for selection of the most optimal LAIP in a given patient. For comparison of MFC MRD values to qPCR values, these were normalized and diagnostic values were set at 1 .

\section{Dilution experiments of in vitro sensitivity of hMICL/CD123 LAIPs}

Dilution experiments were employed to test the theoretical maximal sensitivity in vitro. To this end, BM from two different AML patients were admixed with purified T-lymphocytes from normal donors. Secondly, BM from eight AML patients was admixed with normal BM to approximate the sensitivity of the assay in the clinical setting. In parallel, in five of the patients, in whom at least one molecular marker suitable for quantification by qPCR was present, spiking of RNA from AML cells in normal BM was performed. The dilution ranged from $1: 1$ to $1: 2^{16}$ (in lymphocyte dilutions $1: 1$ to $1: 2^{18}$ ) for each experiment. A minimum of 500,000 events was acquired resulting in a theoretical maximal sensitivity of at least $0 \cdot 004 \%$.

\section{Real-time quantitative PCR}

RNA purification and cDNA synthesis was performed as previously reported (Beillard et al, 2003; Østergaard et al, 2004). Levels of PML-RARA, RUNX1-RUNX1T1, CBFB-MYH11, and WT1 over expression were analysed by TaqMan qPCR on an Mx3000P PCR or Mx3005P PCR thermocycler (Stratagene, La Jolla, CA, USA). All reactions were run on mononuclear cells in triplicates and data normalized to the mean of the two housekeeping genes $\mathrm{c}$-abl oncogene 1, non-receptor tyrosine kinase $(A B L 1)$ and $\beta$-2-microglobulin (B2M). At diagnosis, normalized expression levels were set at one. Primer pairs and probes are given in Table S2. Sensitivities and frequencies of the respective aberrations are given in (Gabert et al, 2003).

\section{Statistics}

The association between $\mathrm{qPCR}$ and MFC values was quantified calculating the Spearman correlation coefficient. The Kaplan-Meier method was used to estimate the distribution of relapse-free survival (RFS), defined as the time between day 1 of treatment and date of relapse, censored at last day of contact. Univariate associations between MRD groups and relapse were tested using log-rank test. Two-tailed $P$-values $<0 \cdot 05$ were considered significant.

\section{Results}

\section{In vitro sensitivity of $h M I C L / C D 123$ LAIPs}

The theoretical sensitivity of the hMICL/CD123 was determined by performing two-fold dilutions of AML cells in highly purified T-cells, the latter acting as true hMICL-/CD123-/ CD34-/CD117- control cells (Fig 2A). The sensitivity of the assay was calculated from the plateau-phase at of the titration curve and was at least 1:4,500 for the hMICL+ CD123 + and at least 1:30,000 for CD34+ hMICL+CD123+ LAIP.

The sensitivity of the hMICL/CD123 assay in a setting resembling that of clinical testing was evaluated. Dilution experiments for $\mathrm{qPCR}$-positive patients are shown in Fig 2B-F, and the three patients without molecular MRD markers are displayed in Fig S1. The detection limits of MFC ranged from $10^{-2}$ to below $10^{-4}$. The spiking LAIP assessments with hMICL/CD123 combinations completely paralleled qPCR quantification for molecularly positive patients. However, for cases with fusion transcript-positive AML the qPCR methods were more sensitive than MFC. On the other hand, in one case (Fig 2B 'empty space') the MFC approach was almost as sensitive as the PML-RARA qPCR assay, reaching below the $10^{-4}$ level. 


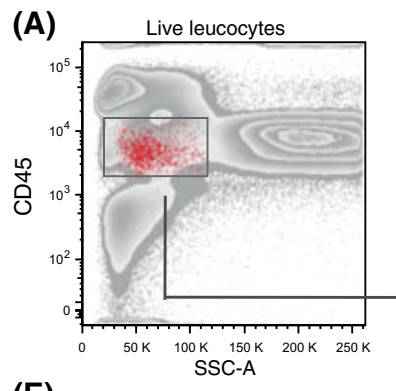

(E) CD45/ow/SSClow/CD14-/CD117+

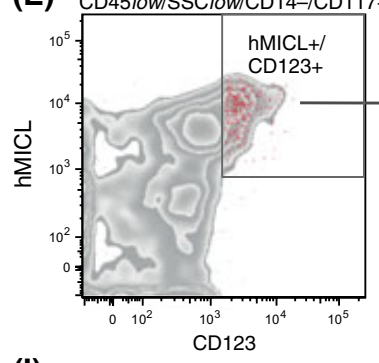

(I) $\mathrm{CD} 45 /$ w/SSClow/CD14-/CD34+

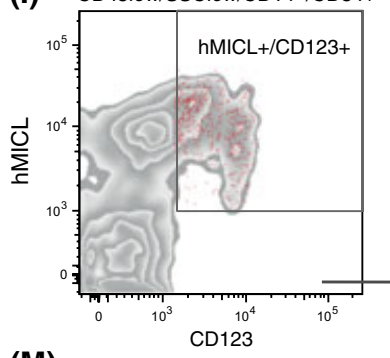

(m)

CD45/ow/SSClow/CD14-/CD34+

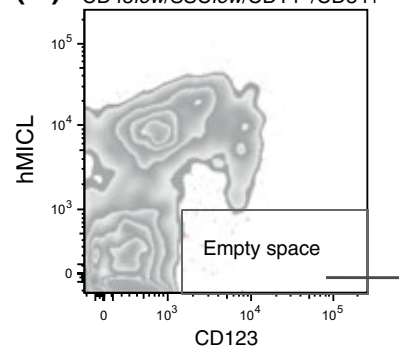

(B)

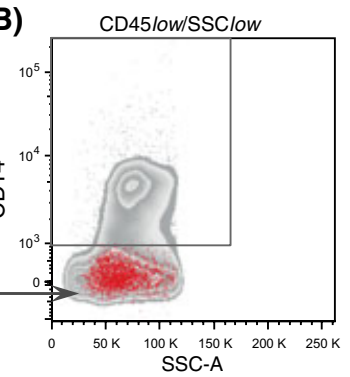

(F)

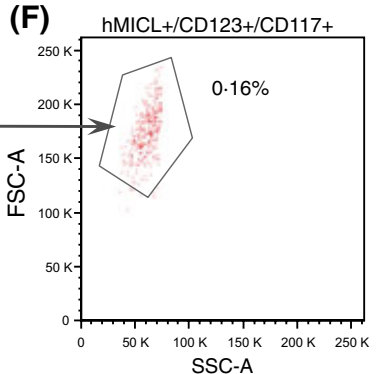

$(\mathrm{J})$

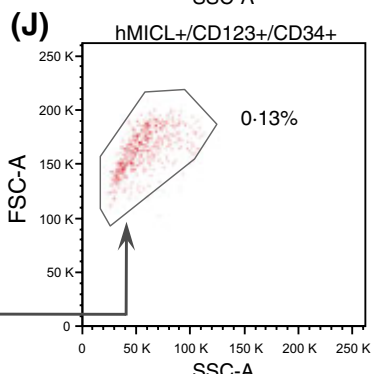

(N)

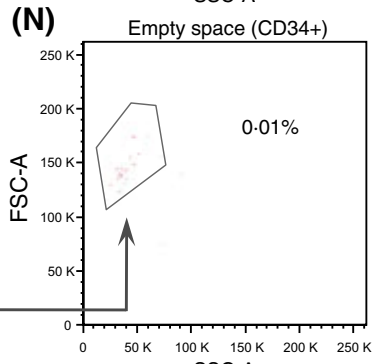

(C)

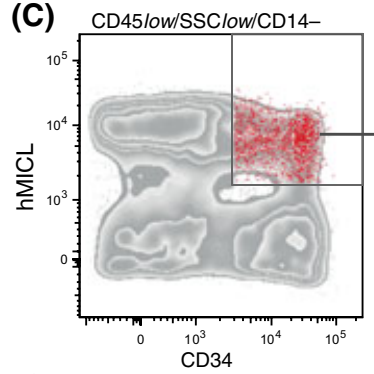

(G) CD45/ow/SSClow/CD14-/CD117+

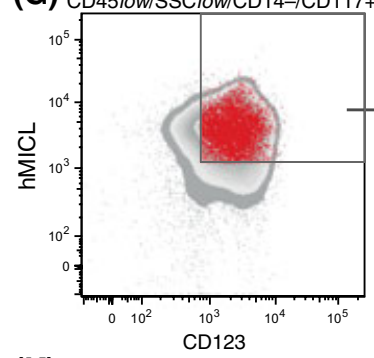

(K) CD45/ow/SSClow/CD14-/CD34+

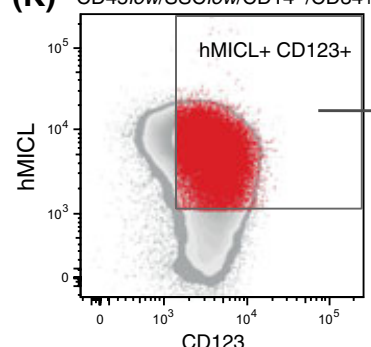

(O) CD45/ow/SSClow/CD14-/CD34+

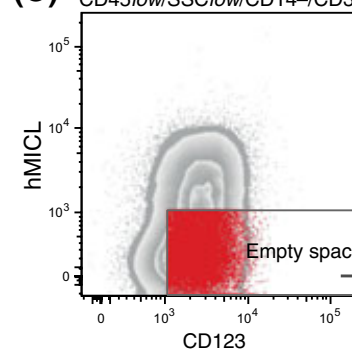

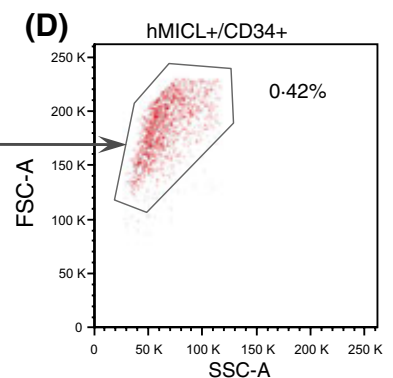

(H)

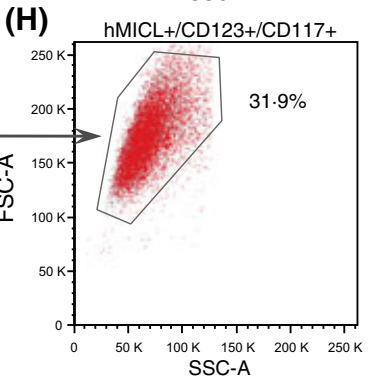

(L)

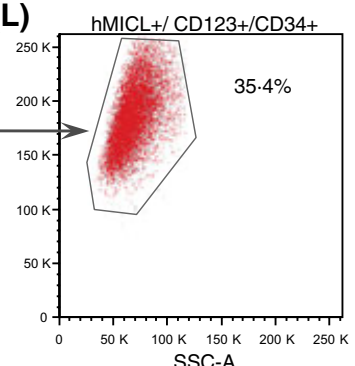

(P)

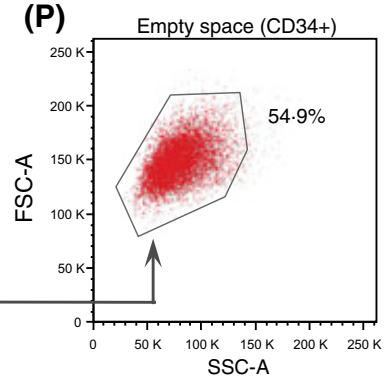

Fig 1. Gating strategy and examples of defined hMICL/CD123 LAIPs. Gating strategy detailed and exemplified in normal bone marrow (BM) and acute myeloid leukaemia (AML) BM samples. The frequencies of leukaemia-associated immunophenotype (LAIP)+ cells within the normal BM are given as median values from all normal samples analysed. Each individual LAIP was analysed in the same way in both AML cases and normal BM. (A) The progenitor population in normal BM and the blast population in AML were characterized by low expression of CD45 and low side scatter (CD45low/SSClow). Granulocytes were excluded based on SSC properties. (B) Monocytes in the CD45low/SSClow were excluded using CD14 staining. (C) hMICL+/CD34+ cells were back-gated into a forward scatter (FSC)/SSC plot in order to ensure homogeneous scatter properties of the population defined (D). Back-gating into a FSC/SSC plot was performed for all LAIPs defined. The $\mathrm{hMICL}+\mathrm{CD} 123+\mathrm{CD} 117+$ population in normal BM (E-F) and in a CD34- AML patient $(\mathrm{G}-\mathrm{H})$. The hMICL+CD123 + CD34 + population in normal BM $(\mathrm{I}+\mathrm{J})$ and in an AML patient $(\mathrm{K}-\mathrm{L})$. The empty space region, defined as CD34+/CD123+/hMICL- cells, in normal BM $(\mathrm{M}+\mathrm{N})$ and in an AML patient $(\mathrm{O}-\mathrm{P})$

Fig. 2. Two-fold dilution series of hMICL+ blasts admixed to T-cells and normal bone marrow with paralleled qPCR results. Dotted lines represent sensitivity of real-time quantitative polymerase chain reaction (qPCR) methods and background of hMICL/CD123 cells from the donor used in each experiment. (A) Titration of hMICL+ cells on a negative background of purified T-cells. Axes in (A) are representative of all experiments A-F. (B) The hMICL/CD123-based leukaemia-associated immunophenotype (LAIP) titrated in a donor with high WT1 background. (C+D) Dilution series of hMICL/CD123-based LAIPs and paralleled qPCR results of PML-RARA and WT1 from two t(15;17) patients. (E-F) Dilution series of hMICL/CD123-based LAIPs and paralleled qPCR results for CBFB-MYH11 + and WT1 from two inv(16) positive patients. ND, normal donor. 
(A)
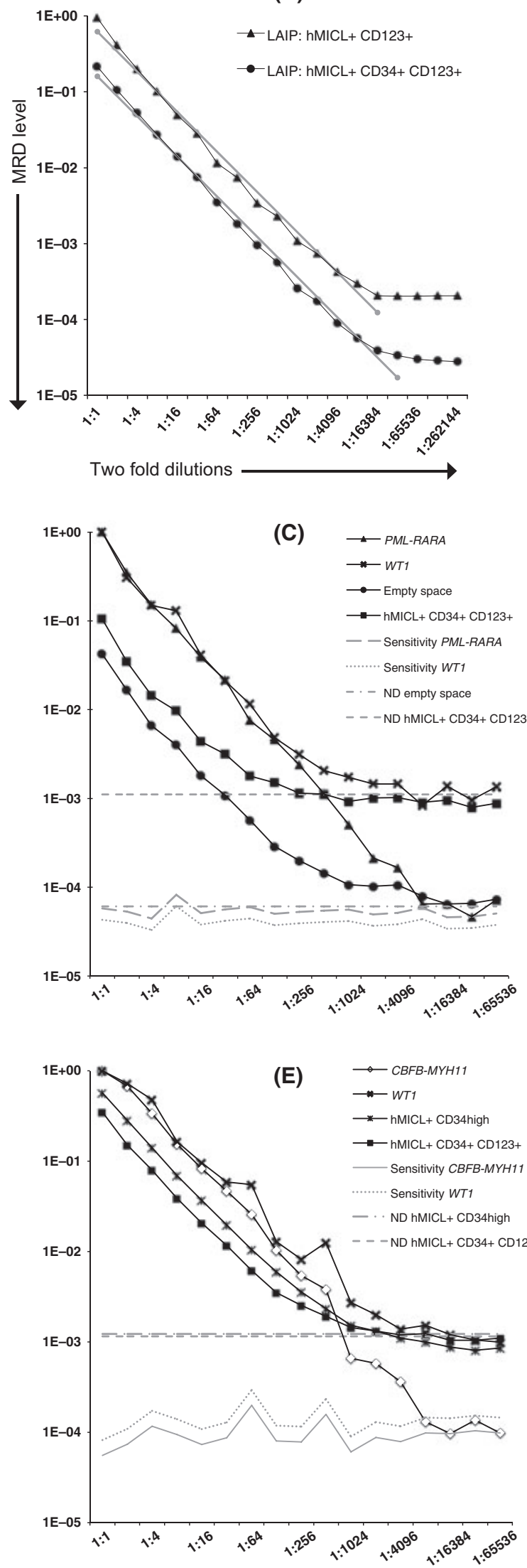

(B)

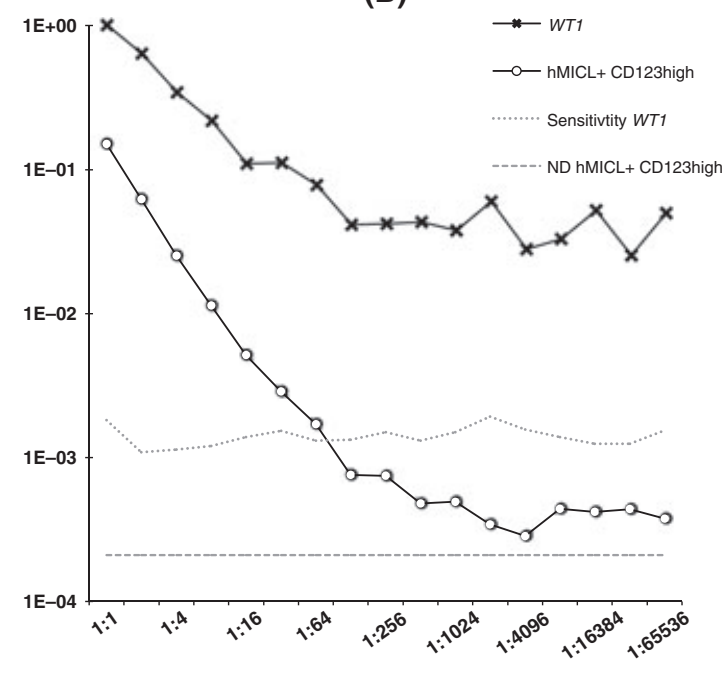

(D)
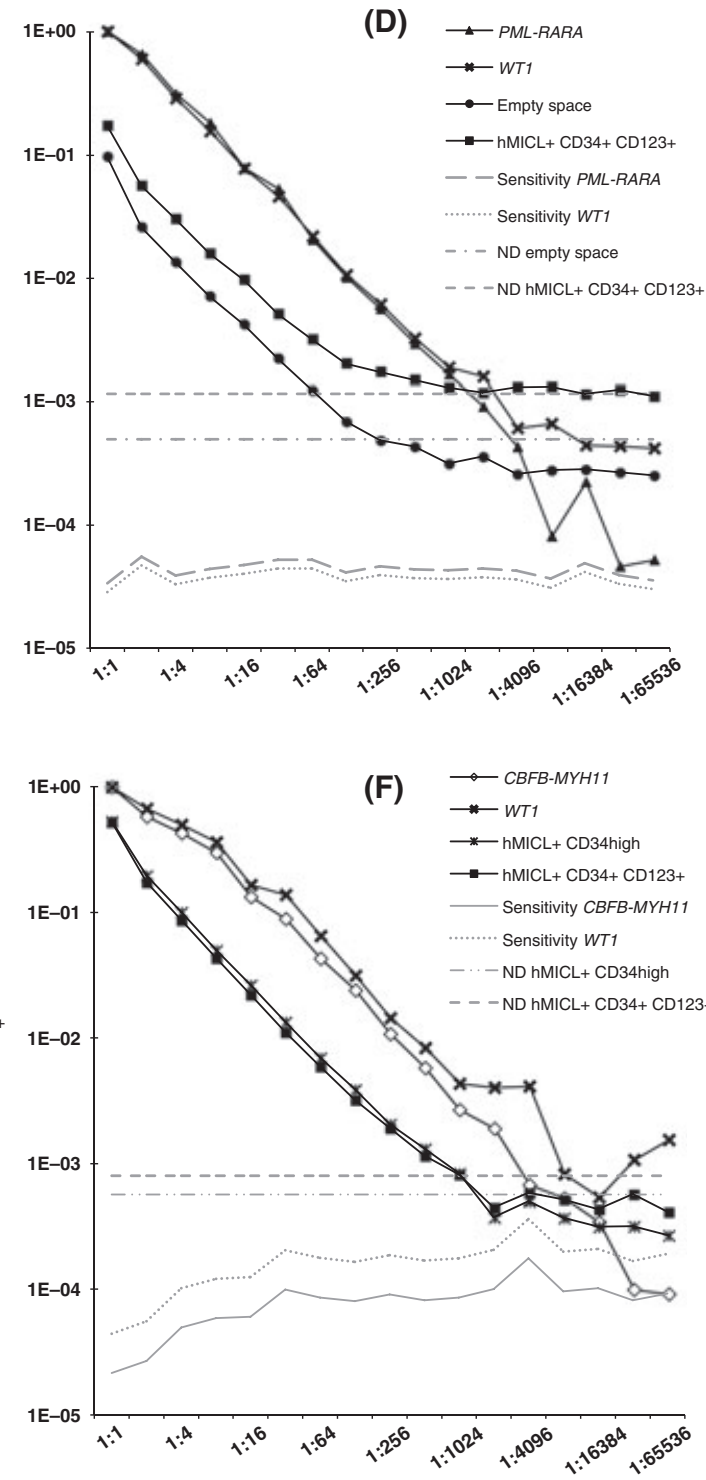
Frequencies of hMICL/CD123-based LAIPs in AML and in normal and regenerating bone marrow

We delineated the value of hMICL/CD123 combinations for MRD detection by comparing BM from AML patients with normal and regenerating BM. The LAIP populations defined, the number of LAIPs identified, and the number of LAIP positive patients by application of the hMICL/CD123 to the CD45/CD34/CD117 backbone are detailed in Table II. A total of 11 populations fulfilling LAIP criteria were identified in 64 of the total cohort of 69 patients (93\%) at diagnosis. In fact, because more than one highly sensitive LAIP could be identified in some patients, a total of 150 LAIPs in 62 positive cases were defined. On the other hand, we failed to identify a LAIP in five patients (7\%). We successfully applied the 'empty space' concept in 12 patients. The most frequent LAIPs were CD34+hMICL+, hMICL + CD $34^{\text {high }}$ and CD123 + CD34+ (Table II).

\section{Quantitation of differences in hMICL/CD123-based LAIP-positive cells between AML and normal and regenerating $B M$ samples}

A crucial issue in the optimization of a routine MRD marker is the delineation of differences between leukaemic and normal and regenerating BM cells. We approached this issue by employing the LD concept (Table II). As will appear, the maximum LD from the median frequency in both normal and regenerating $\mathrm{BM}$ allowed for identification of the three most optimal LAIPs in the series. For normal BM these were: hMICL+CD $123^{\text {high }}$, Empty space $(\mathrm{CD} 34+\mathrm{AML})$, and $\mathrm{hMICL}+\mathrm{CD} 34^{\text {high }}$ and in regenerating BM: Empty space $(\mathrm{CD} 34+\mathrm{AML}), \quad \mathrm{hMICL}+\mathrm{CD} 123^{\text {high }}$, and empty space (CD34- AML) in that order of maximal LD.

\section{Comparison of MRD as measured by $q P C R$ and by hMICL/CD123 based MCF post induction}

We directly compared MRD by the hMICL/CD123 assay and as gold standard -optimized qPCR assays (Fig 3). Here, 21 molecularly positive patients were evaluable for MRD postinduction, three positive for a fusion transcript and 18 patients had WT1 over expression. Relative MRD levels of the most sensitive hMICL/CD123 LAIPs and standard MFC LAIPs were compared separately to relative qPCR MRD levels. The correlation between our standard MFC LAIPs (Table S1) and qPCR was $r=0.574(P=0.0065)$ and even stronger for the hMICL/ CD123 based assay $r=0.676(P=0.008)$.

\section{Level of hMICL/CD123 as a prognostic test at first CR evaluation}

The level of LAIP post-induction was evaluated as a marker for treatment response. When tabulating the levels of hMICL/CD123 LAIPs, we found that of 13 patients with
hMICL/CD123 MRD levels above the median in regenerating BM, 10 patients relapsed. Conversely, only one patient with hMICL/CD123 MRD below the median in regenerating BM relapsed (Fig 4). With an admittedly short period of observation, a statistically significant difference $(P<0.001)$ in RFS was detected, with patients with hMICL/CD123 LAIPs levels below the median in regenerating BM less prone to relapse.

\section{Validation of longitudinal monitoring of $M R D$ by hMICL/CD123 LAIPS in comparison to qPCR}

To determine the value of the hMICL/CD123 MRD marker in the follow-up phase, we monitored 15 patients longitudinally (median number of MRD samplings $=3$, range 3-6) by relative hMICL/CD123-based MRD by MFC and compared these results directly to relative qPCR MRD. We found that these widely different methodologies yielded closely paralleled MRD curves and kinetics (Fig 5).

\section{Discussion}

We have previously reported that the hMICL and CD123 antigens were widely expressed and stable markers in more than $90 \%$ of AML samples. This contrasted to other surface proteins routinely employed for AML immunophenotyping, because these were not only less frequent at diagnosis but also prone to change at relapse in up to $35 \%$ of cases (Oelschlägel et al, 2000; Voskova et al, 2004; Larsen et al, 2012). Moreover, as both hMICL and CD123 have been suggested as strong markers for LSCs (Jordan et al, 2000; van Rhenen et al, 2007b; Jin et al, 2009), we have pursued the issue of the hMICL/CD123 markers in combination with CD45 and the stem cell antigens CD34 and CD117 as an MFC MRD tool. Through spiking experiments and by testing a prospective patient cohort, we showed that this combination of MoAbs constituted a simple, widely applicable MFC MRD tool. Regarding the specificity of the LAIPs, high hMICL/CD123 MRD levels post-induction were strongly predictive of relapse.

The CD34 antigen is a widely applied backbone in LAIP identification due to its expression on immature cells. However, CD34 negativity of diagnostic samples has been reported as high as $20-30 \%$ when employing a cut-off of $1 \%$ (Al-Mawali et al, 2009; Taussig et al, 2010). In our series of patients, $18 / 69$ (26\%) were classified as CD34- using this threshold. Among those patients, we only failed to identify a LAIP for MRD detection in one patient when applying the hMICL/CD123-based approach. Thus, this combination of antibodies paves the way for improved LAIP characterization and MRD quantification by MFC in the otherwise poorly described CD34- AML cases. Moreover, hMICL/CD123 immunophenotyping may be taken advantage of in cell sorting experiments followed by molecular and cytogenetic analyses and functional studies to better characterize LSCs in CD34AML. On-going molecular studies of FACS (fluorescent-activated cell sorting) sorted hMICL/CD123 LAIP-positive cells 


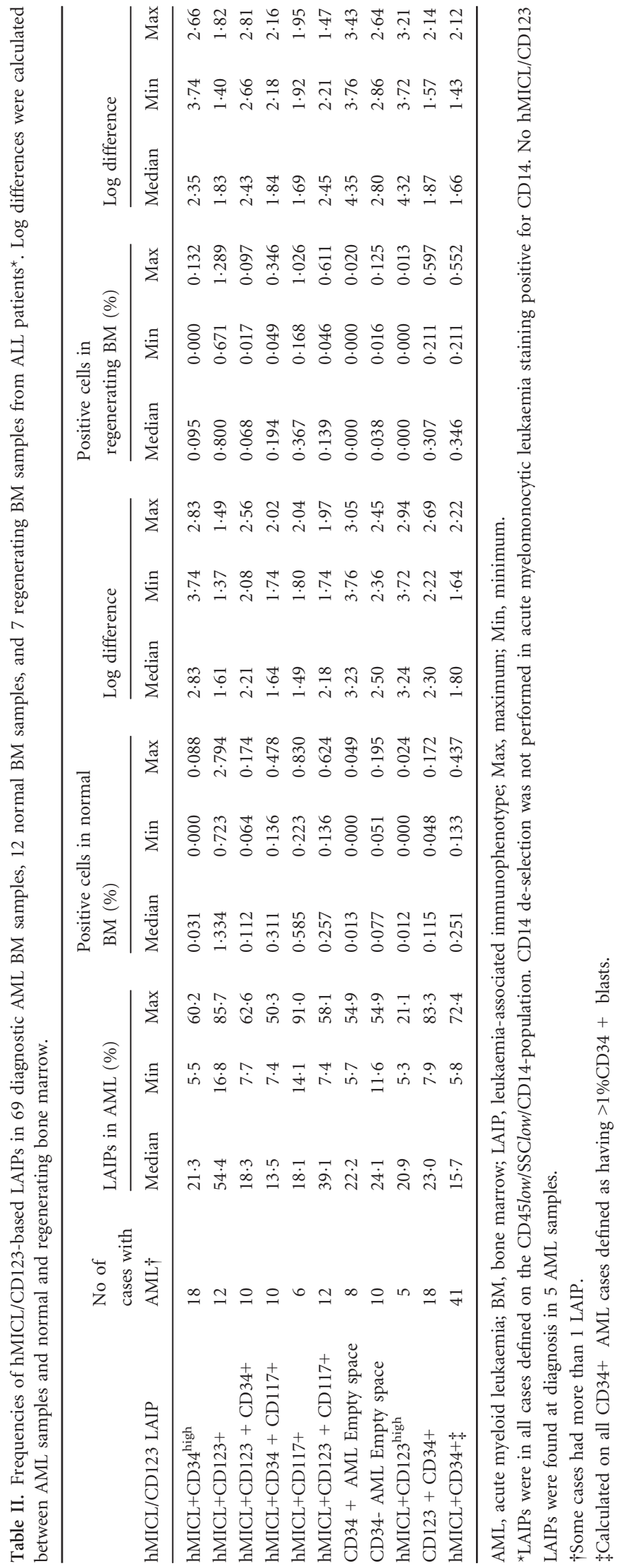




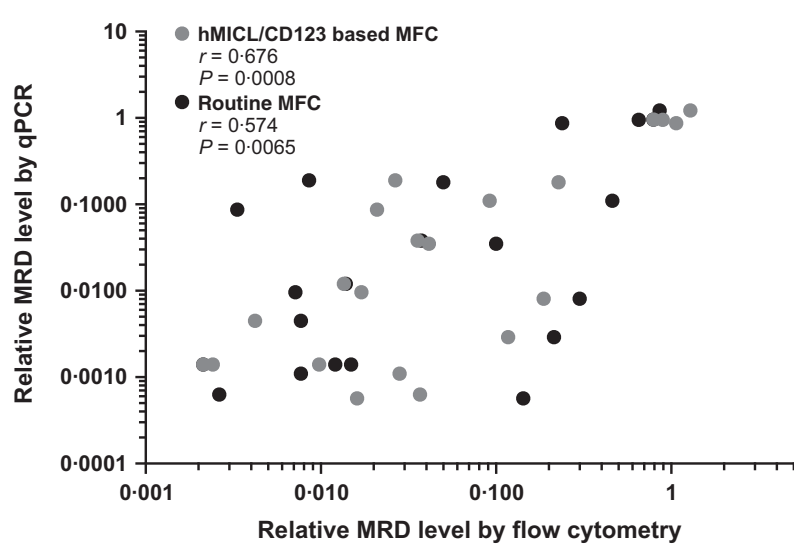

Fig 3. Comparison of relative minimal residual disease (MRD) values as measured by real-time quantitative polymerase chain reaction (qPCR) and multicolour flow cytometry (MFC) at post-induction time points.

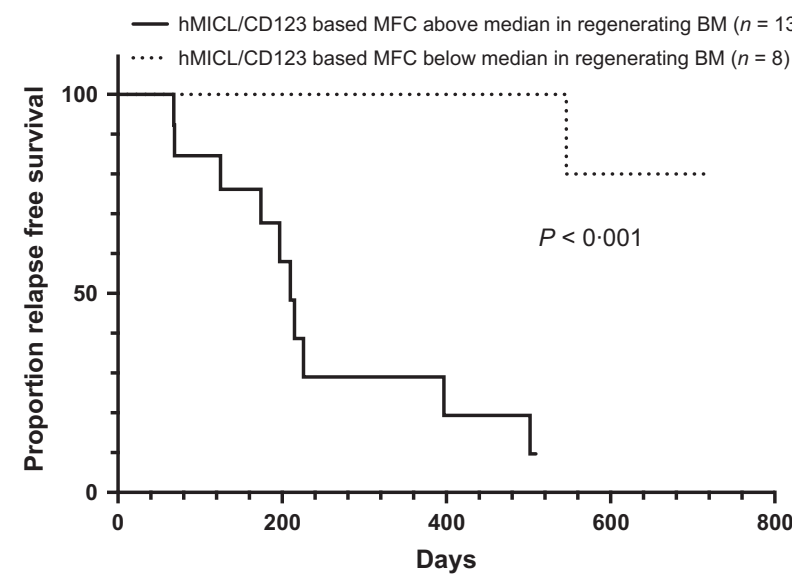

Fig 4. Kaplan-Meier plot of relapse-free survival of patients stratified at post-induction evaluation according to $\mathrm{h}$ MICL/CD123 expression. Patients were divided according to hMICL/CD123; patients with high levels display an unfavourable outcome $(P<0.001$, Log rank test). MFC, multicolour flow cytometry.

in our laboratory have confirmed that these populations are, in fact, highly enriched for AML-related molecular aberrations (data not shown).

In total, the search for LAIPs was positive in $93 \%$ of diagnostic AML samples when using the hMICL/CD123-based combinations, with sensitivities down to $10^{-4}$. By inference, the addition of more colours should undoubtedly allow for further increased sensitivity and applicability, and reduce the amount of BM necessary, as well as costs incurred and time spent. At least four things should be kept in mind: Firstly, the amount of labour and reagents using the present approach are much less than the traditional one, which involves multiple antibody combinations (Kern et al, 2010b; Béné et al, 2011; van Dongen et al, 2012). Secondly, in routine MFC MRD assessment, it is recommended to determine at least two independent LAIPs to circumvent the problem of false negative results due to immunophenotypic shifts (Kern et al, 2010a). As both hMICL and CD123 can be detected with conserved antigen densities at relapse, this may well allow for reducing the number of MoAbs and tubes used (Langebrake et al, 2005; Larsen et al, 2012). Thirdly, our data have pinpointed the potential importance of applying the empty space - also termed the 'different-from-normal' concept (Lucio et al, 2001; Paietta, 2012a). While highly sensitive in some of our patients, more are, nevertheless, needed to conclusively determine the impact of empty space hMICL/ CD123 LAIPs in AML. Finally, the hMICL/CD123 assessment is based on the detection of antigens associated with LSCs (Majeti, 2010; Estey, 2012). This could lend an added bonus of the approach due to its possible marking of cells thought to selectively survive anti-leukaemia treatment. In fact, standard MFC panels may fail to detect persisting LSCs posttherapy, which may explain treatment failure in otherwise MFC MRD-negative patients (Bachas et al, 2012; Buccisano et al, 2012).

It might be argued that, because LSC-directed MRD quantification by MFC does not include all malignant cells, this could lead to a lowering of sensitivity in terms of using LD calculations in the search for the most optimal LAIP. This was evidenced in our longitudinally tabulated $\log$ changes of MRD in paralleled relative $\mathrm{qPCR}$ and $\mathrm{MFC}$ analyses. While we did observe a striking parallelism, qPCR performed better than MFC in this setting. However, this does not in any way diminish the importance of the LSC MFC assays, as the one presented here. In fact, the LSC assays' higher specificity and improved sensitivity due to low background expression may offer a significant advantage (van Rhenen et al, 2005; ten Cate et al, 2010). Different MRD approaches are compared based on their sensitivity, specificity and applicability, and results are reported as continuous variables that may not be directly comparable (e.g. log changes versus percentages of LAIP+ cells) (Paietta, 2012a). Irrespective of this, tandem performance and comparison of these methodologies will ensure better MRD coverage and facilitate the establishment of method-specific thresholds and the translation of MRD results into clinical decision making.

The assessment of MRD is a surrogate marker of treatment efficacy that has prognostic impact, although formal implementation of MRD assessment in the management of AML patients is still in its infancy (Hourigan \& Karp, 2013). In addition, the preferred platform for the measurement of MRD is still a matter for debate. We have recently suggested a context-driven application of MRD-methods (Hokland et al, 2012). The MFC assays have the advantages of wide applicability and short turnover time, and enable the exact enumeration of LAIP-positive cells. On the other hand, the fusion transcripts and somatically mutated genes are the most sensitive and specific MRD targets and can, together with overexpressed genes, be applied for 

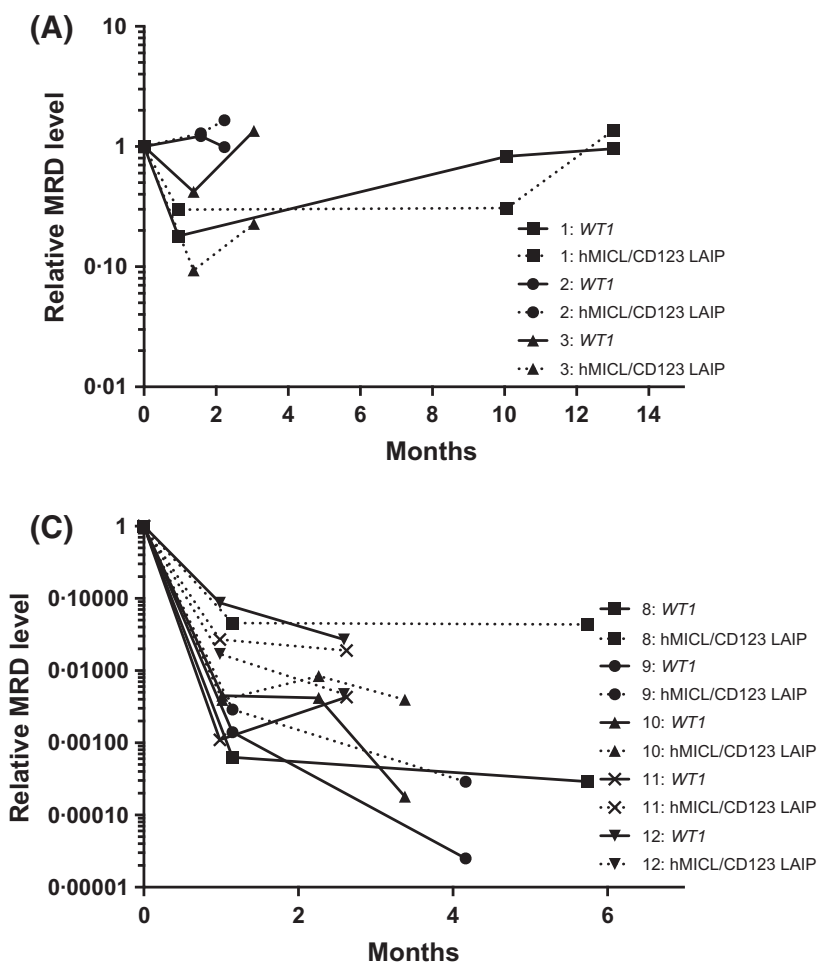
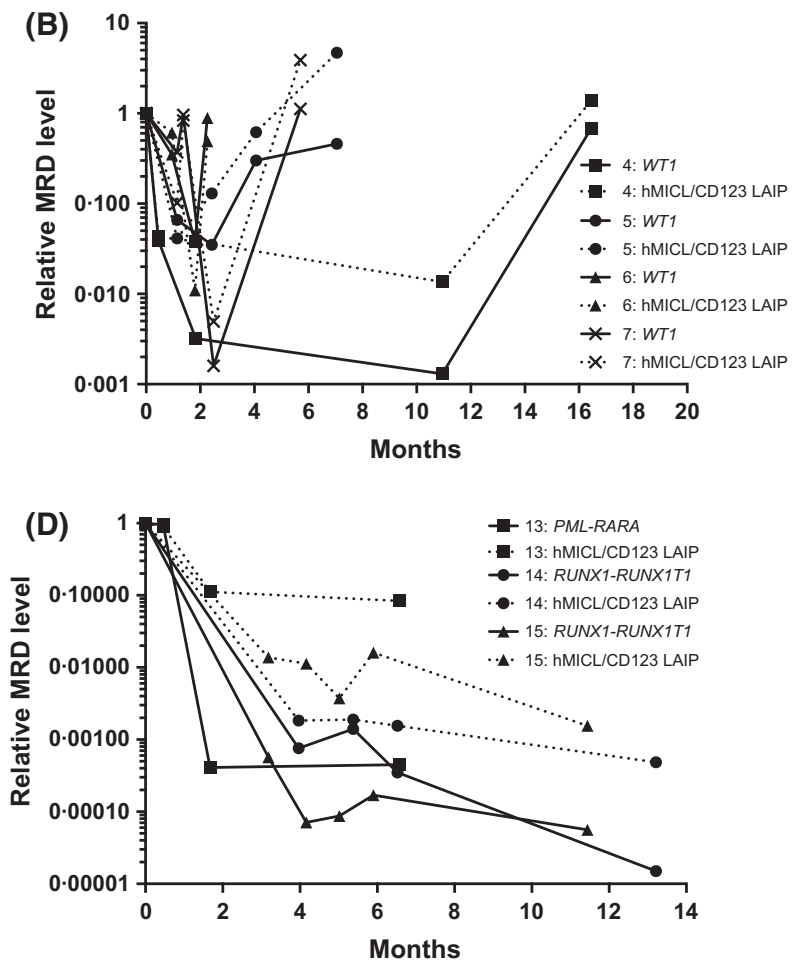

Fig 5. Longitudinal registered log changes in MRD in paralleled MFC and qPCR evaluation. Paralleled log changes of WT1 over expression measured by real-time quantitative polymerase chain reaction (qPCR) and of multicolour flow cytometry (MFC) hMICL/CD123 leukaemia-associated immunophenotypes (LAIPs) in (A) patients not attaining haematological complete remission (CR),(B) patients achieving haematological CR after first induction followed by a later relapse, (C)patients in haematological CR at the time of reporting, and (D) paralleled qPCR and MFC minimal residual disease $(\mathrm{MRD})$ results in three fusion transcript-positive patients in haematological CR.

monitoring MRD by qPCR. Additionally, new methods, such as next generation sequencing, are fast gaining ground (Kohlmann et al, 2013). How then to implement the hMICL/CD123-based assay in a routine clinical setting? At the present time this assay should not replace standard LAIP marking. Although the sensitivity and applicability of the assay is established, prospective evaluation in larger patient cohorts are needed to further solidify its performance, and establish clinical cut-off values and recommendations on sampling frequencies.

In conclusion, we have defined an MFC assay employing hMICL/CD123 in combination with CD34 and CD117, which is comparable to qPCR. It should allow for simpler, faster and more standardized MRD determination by MFC than hitherto described assays due to LAIP coverage of more than $90 \%$ of the patients with sensitivities down to $10^{-4}$ and improve MRD assessment in CD34- AML.

\section{Acknowledgements}

The work was supported by grants to $\mathrm{PH}$ from The Danish Cancer Society, The Danish MRC, The John and Birthe Meyer Foundation, and the Karen Elise Jensen Foundation. GB has received funding from The Wellcome Trust. We thank our patients for contributing samples, and for continuous input during these efforts.

\section{Author contributions}

ASR, HØL and PH designed the study. ASR, LN, CGN, and $\mathrm{HBO}$ analysed the data. ASR wrote first draft of the manuscript. GB and TJ contributed reagents. All authors made significant contributions to, reviewed, and approved the final version of the manuscript.

\section{Conflict-of-interest disclosure}

TJ is director at Dako. GB receives royalty payments from the sale of the anti-hMICL antibody by eBioscience. All other authors declare no conflicts of interest.

\section{Supporting Information}

Additional Supporting Information may be found in the online version of this article:

Fig S1. Spiking experiments of three AML samples without molecular qPCR handle for MRD quantification.

Table S1. Monoclonal antibodies used for standard immunostaining of diagnostic AML samples and for MRD evaluation.

Table S2. Primer and probe sequences for qPCR amplification found in the core binding factor leukemias and of WT1. 


\section{References}

Al-Mawali, A., Gillis, D., Hissaria, P. \& Lewis, I. (2008) Incidence, sensitivity, and specificity of leukemia-associated phenotypes in acute myeloid leukemia using specific five-color multiparameter flow cytometry. American Journal of Clinical Pathology, 129, 934-945.

Al-Mawali, A., Gillis, D. \& Lewis, I. (2009) The role of multiparameter flow cytometry for detection of minimal residual disease in acute myeloid leukemia. American Journal of Clinical Pathology, 131, 16-26.

Bachas, C., Schuurhuis, G.J., Assaraf, Y.G., Kwidama, Z.J., Kelder, A., Wouters, F., Snel, A.N., Kaspers, G.J.L. \& Cloos, J. (2012) The role of minor subpopulations within the leukemic blast compartment of AML patients at initial diagnosis in the development of relapse. Leukemia, 26, 1313-1320.

Beillard, E., Pallisgaard, N., van der Velden, V.H.J., Bi, W., Dee, R., van der Schoot, E., Delabesse, E., Macintyre, E., Gottardi, E., Saglio, G., Watzinger, F., Lion, T., van Dongen, J.J.M., Hokland, P. \& Gabert, J. (2003) Evaluation of candidate control genes for diagnosis and residual disease detection in leukemic patients using 'real-time' quantitative reverse-transcriptase polymerase chain reaction (RQ-PCR) - a Europe against cancer program. Leukemia, 17, 2474-2486.

Béné, M.C., Nebe, T., Bettelheim, P., Buldini, B., Bumbea, H., Kern, W., Lacombe, F., Lemez, P., Marinov, I., Matutes, E., Maynadié, M., Oelschlagel, U., Orfao, A., Schabath, R., Solenthaler, M., Tschurtschenthaler, G., Vladareanu, A.M., Zini, G., Faure, G.C. \& Porwit, A. (2011) Immunophenotyping of acute leukemia and lymphoproliferative disorders: a consensus proposal of the European LeukemiaNet Work Package 10. Leukemia, 25, 567-574.

Bonnet, D. (2001) Normal and leukemic CD34negative human hematopoietic stem cells. Reviews in Clinical and Experimental Hematology, 5, 42-61.

Borowitz, M.J., Guenther, K.L., Shults, K.E. \& Stelzer, G.T. (1993) Immunophenotyping of acute leukemia by flow cytometric analysis. Use of CD45 and right-angle light scatter to gate on leukemic blasts in three-color analysis. American Journal of Clinical Pathology, 100, 534-540.

Buccisano, F., Maurillo, L., Spagnoli, A., Del Principe, M.I., Ceresoli, E., Lo Coco, F., Arcese, W., Amadori, S. \& Venditti, A. (2009) Monitoring of minimal residual disease in acute myeloid leukemia. Current Opinion in Oncology, 21, 582-588.

Buccisano, F., Maurillo, L., Del Principe, M.I., Del Poeta, G., Sconocchia, G., Lo-Coco, F., Arcese, W., Amadori, S. \& Venditti, A. (2012) Prognostic and therapeutic implications of minimal residual disease detection in acute myeloid leukemia. Blood, 119, 332-341.

ten Cate, B., de Bruyn, M., Wei, Y., Bremer, E. \& Helfrich, W. (2010) Targeted elimination of leukemia stem cells; a new therapeutic approach in hemato-oncology. Current Drug Targets, 11, 95-110.

Dinardo, C.D. \& Luger, S.M. (2012) Beyond morphology: minimal residual disease detection in acute myeloid leukemia. Current Opinion in Hematology, 19, 82-88.

van Dongen, J.J.M., Lhermitte, L., Böttcher, S., Almeida, J., van der Velden, V.H.J., Flores-Montero, J., Rawstron, A., Asnafi, V., Lécrevisse, Q., Lucio, P., Mejstrikova, E., Szczepański, T., Kalina, T., de Tute, R., Brüggemann, M., Sedek, L., Cullen, M., Langerak, A.W., Mendonça, A., Macintyre, E., Martin-Ayuso, M., Hrusak, O., Vidriales, M.B. \& Orfao, A. (2012) EuroFlow antibody panels for standardized n-dimensional flow cytometric immunophenotyping of normal, reactive and malignant leukocytes. Leukemia, 26, 1908-1975.

Estey, E.H. (2012) Acute myeloid leukemia: 2012 update on diagnosis, risk stratification, and management. American Journal of Hematology, 87, 89-99.

Estey, E. \& Döhner, H. (2006) Acute myeloid leukaemia. Lancet, 368, 1894-1907.

Feller, N., van der Pol, M.A., van Stijn, A., Weijers, G.W.D., Westra, A.H., Evertse, B.W., Ossenkoppele, G.J. \& Schuurhuis, G.J. (2004) MRD parameters using immunophenotypic detection methods are highly reliable in predicting survival in acute myeloid leukaemia. Leukemia, 18, 1380-1390.

Gabert, J., Beillard, E., van der Velden, V.H.J., Bi, W., Grimwade, D., Pallisgaard, N., Barbany, G., Cazzaniga, G., Cayuela, J.M., Cavé, H., Pane, F., Aerts, J.L.E., De Micheli, D., Thirion, X., Pradel, V., González, M., Viehmann, S., Malec, M., Saglio, G. \& van Dongen, J.J.M. (2003) Standardization and quality control studies of 'real-time' quantitative reverse transcriptase polymerase chain reaction of fusion gene transcripts for residual disease detection in leukemia - a Europe Against Cancer program. Leukemia, 17, 2318-2357.

Grimwade, D., Jovanovic, J.V., Hills, R.K., Nugent, E.A., Patel, Y., Flora, R., Diverio, D., Jones, K., Aslett, H., Batson, E., Rennie, K., Angell, R., Clark, R.E., Solomon, E., Lo-Coco, F., Wheatley, K. \& Burnett, A.K. (2009) Prospective minimal residual disease monitoring to predict relapse of acute promyelocytic leukemia and to direct preemptive arsenic trioxide therapy. Journal of Clinical Oncology, 27, 3650-3658.

Grimwade, D., Vyas, P. \& Freeman, S. (2010a) Assessment of minimal residual disease in acute myeloid leukemia. Current Opinion in Oncology, 22, 656-663.

Grimwade, D., Hills, R.K., Moorman, A.V., Walker, H., Chatters, S., Goldstone, A.H., Wheatley, K., Harrison, C.J., Burnett, A.K.; National Cancer Research Institute Adult Leukaemia Working Group (2010b) Refinement of cytogenetic classification in acute myeloid leukemia: determination of prognostic significance of rare recurring chromosomal abnormalities among 5876 younger adult patients treated in the United Kingdom Medical Research Council trials. Blood, 116, 354-365.

Hokland, P., Ommen, H.B., Nyvold, C.G. \& Roug, A.S. (2012) Sensitivity of minimal residual disease in acute myeloid leukaemia in first remission-methodologies in relation to their clinical situation. British Journal of Haematology, 158, 569-580.

Hourigan, C.S. \& Karp, J.E. (2013) Minimal residual disease in acute myeloid leukaemia. Nature reviews. Clinical Oncology, 10, 460-471.

Jin, L., Lee, E., Ramshaw, H., Busfield, S., Peoppl, A., Wilkinson, L., Guthridge, M.A., Thomas, D., Barry, E.F., Boyd, A., Gearing, D.P., Vairo, G., Lopez, A.F., Dick, J.E. \& Lock, R.B. (2009) Monoclonal antibody-mediated targeting of CD123, IL-3 receptor alpha chain, eliminates human acute myeloid leukemic stem cells. Cell Stem Cell, 5, 31-42.

Jordan, C.T., Upchurch, D., Szilvassy, S.J., Guzman, M.L., Howard, D.S., Pettigrew, A.L., Meyerrose, T., Rossi, R., Grimes, B., Rizzieri, D.A., Luger, S.M. \& Phillips, G.L. (2000) The interleukin-3 receptor alpha chain is a unique marker for human acute myelogenous leukemia stem cells. Leukemia, 14, 1777-1784

Kern, W., Bacher, U., Haferlach, C., Schnittger, S. \& Haferlach, T. (2010a) The role of multiparameter flow cytometry for disease monitoring in AML. Best Practice \& Research Clinical Haematology, 23, 379-390.

Kern, W., Haferlach, C., Schnittger, S. \& Haferlach, T. (2010b) Clinical utility of multiparameter flow cytometry in the diagnosis of 1013 patients with suspected myelodysplastic syndrome: correlation to cytomorphology, cytogenetics, and clinical data. Cancer, 116, 4549-4563.

Kohlmann, A., Grossmann, V., Nadarajah, N. \& Haferlach, T. (2013) Next-generation sequencing - feasibility and practicality in haematology. British journal of haematology, 160, 736-753.

Lacombe, F., Durrieu, F., Briais, A., Dumain, P., Belloc, F., Bascans, E., Reiffers, J., Boisseau, M.R. \& Bernard, P. (1997) Flow cytometry CD45 gating for immunophenotyping of acute myeloid leukemia. Leukemia, 11, 1878-1886.

Langebrake, C., Brinkmann, I., Teigler-Schlegel, A., Creutzig, U., Griesinger, F., Puhlmann, U. \& Reinhardt, D. (2005) Immunophenotypic differences between diagnosis and relapse in childhood AML: Implications for MRD monitoring. Cytometry. Part B, Clinical Cytometry, 63, 1-9.

Larsen, H.Ø., Roug, A.S., Just, T., Brown, G.D. \& Hokland, P. (2012) Expression of the hMICL in acute myeloid leukemia-a highly reliable disease marker at diagnosis and during follow-up. Cytometry. Part B, Clinical Cytometry, 82, 3-8.

Lucio, P., Gaipa, G., van Lochem, E.G., van Wering, E.R., Porwit-MacDonald, A., Faria, T., Bjorklund, E., Biondi, A., van den Beemd, 
M.W., Baars, E., Vidriales, B., Parreira, A., van Dongen, J.J., San Miguel, J.F., Orfao, A.; BIOMED-I (2001) BIOMED-I concerted action report: flow cytometric immunophenotyping of precursor B-ALL with standardized triple-stainings. BIOMED-1 Concerted Action Investigation of Minimal Residual Disease in Acute Leukemia: International Standardization and Clinical Evaluation. Leukemia, 15, 1185-1192.

Majeti, R. (2010) Monoclonal antibody therapy directed against human acute myeloid leukemia stem cells. Oncogene, 30, 1009-1019.

Oelschlägel, U., Nowak, R., Schaub, A., Köppel, C., Herbst, R., Mohr, B., Löffler, C., Range, U., Günther, H., Assmann, M., Siegert, E., Wendt, E., Huhn, R., Bräutigam, E. \& Ehninger, G. (2000) Shift of aberrant antigen expression at relapse or at treatment failure in acute leukemia. Cytometry, 42, 247-253.

Ossenkoppele, G.J., van de Loosdrecht, A.A. \& Schuurhuis, G.J. (2011) Review of the relevance of aberrant antigen expression by flow cytometry in myeloid neoplasms. British Journal of Haematology, 153, 421-426.

Østergaard, M., Olesen, L.H., Hasle, H., Kjeldsen, E. \& Hokland, P. (2004) WT1 gene expression: an excellent tool for monitoring minimal residual disease in $70 \%$ of acute myeloid leukaemia patients - results from a single-centre study. British Journal of Haematology, 125, 590-600.
Paietta, E. (2012a) Minimal residual disease in acute myeloid leukemia: coming of age. Hematology/The Education Program of the American Society of Hematology. American Society of Hematology. Education Program, 2012, 35-42.

Paietta, E. (2012b) When it comes to MRD, AML $\neq$ ALL. Blood, 120, 1536-1537.

van der Pol, M.A., Feller, N., Roseboom, M., Moshaver, B., Westra, G., Broxterman, H.J., Ossenkoppele, G.J. \& Schuurhuis, G.J. (2003) Assessment of the normal or leukemic nature of CD34 + cells in acute myeloid leukemia with low percentages of CD34 cells. Haematologica, 88, 983-993.

van Rhenen, A., Feller, N., Kelder, A., Westra, A., Rombouts, E., Zweegman, S., van der Pol, M., Waisfisz, Q., Ossenkoppele, G. \& Schuurhuis, G. (2005) High stem cell frequency in acute myeloid leukemia at diagnosis predicts high minimal residual disease and poor survival. Clinical Cancer Research, 11, 6520-6527.

van Rhenen, A., Moshaver, B., Kelder, A., Feller, N., Nieuwint, A.W.M., Zweegman, S., Ossenkoppele, G.J. \& Schuurhuis, G.J. (2007a) Aberrant marker expression patterns on the CD34 + CD38- stem cell compartment in acute myeloid leukemia allows to distinguish the malignant from the normal stem cell compartment both at diagnosis and in remission. Leukemia, 21, 1700-1707. van Rhenen, A., van Dongen, G.A.M.S., Kelder, A., Rombouts, E.J., Feller, N., Moshaver, B., Stigtervan Walsum, M., Zweegman, S., Ossenkoppele, G.J. \& Schuurhuis, G.J. (2007b) The novel AML stem cell associated antigen CLL-1 aids in discrimination between normal and leukemic stem cells. Blood, 110, 2659-2666.

Taussig, D.C., Vargaftig, J., Miraki-Moud, F., Griessinger, E., Sharrock, K., Luke, T., Lillington, D., Oakervee, H., Cavenagh, J., Agrawal, S.G., Lister, T.A., Gribben, J.G. \& Bonnet, D. (2010) Leukemia-initiating cells from some acute myeloid leukemia patients with mutated nucleophosmin reside in the CD34(-) fraction. Blood, 115, 1976-1984.

Vora, A., Goulden, N., Wade, R., Mitchell, C., Hancock, J., Hough, R., Rowntree, C. \& Richards, S. (2013) Treatment reduction for children and young adults with low-risk acute lymphoblastic leukaemia defined by minimal residual disease (UKALL 2003): a randomised controlled trial. The Lancet Oncology, 14, 199-209.

Voskova, D., Schoch, C., Schnittger, S., Hiddemann, W., Haferlach, T. \& Kern, W. (2004) Stability of leukemia-associated aberrant immunophenotypes in patients with acute myeloid leukemia between diagnosis and relapse: comparison with cytomorphologic, cytogenetic, and molecular genetic findings. Cytometry. Part B, Clinical cytometry, 62, $25-38$. 